\title{
Study of the field emitted by a source placed inside a two-dimensional left-handed metamaterial
}

\author{
Irfan Bulu, Humeyra Caglayan, Koray Aydin, and Ekmel Ozbay \\ Nanotechnology Research Center, Department of Physics, Department of Electrical and Electronics Engineering, \\ Bilkent, 06800 Ankara, Turkey
}

Received October 6, 2006; revised December 14, 2006; accepted December 15, 2006; posted January 4, 2007 (Doc. ID 75847); published March 5, 2007

\begin{abstract}
We studied the properties of electromagnetic waves that were emitted from a source placed inside a lefthanded medium based on a two-dimensional labyrinth. While the arguments of geometrical optics suggest that the field emitted from the source would be focused outside the left-handed medium no matter where the source was placed, our results proved the contrary. We found that the field emitted from the source was focused outside the left-handed medium when the source was placed inside the medium at a certain distance away from the interface. Moreover, our results showed that the field emitted from the source was focused on the subwavelength dimensions outside the left-handed medium. () 2007 Optical Society of America

OCIS codes: $350.4010,350.5500$.
\end{abstract}

It was back in the 1960s that the peculiar properties of a medium with simultaneously negative permittivity and permeability were predicted. ${ }^{1}$ Negative refraction, the reversal of the Doppler shift, and subwavelength resolution are among these peculiar properties. $^{2-4}$ Simultaneous negative permittivity and permeability were recently realized in the form of a periodic arrangement of thin wires and resonant magnetic inclusions, such as split-ring resonators or labyrinth structures. ${ }^{5,6}$ This new class of artificial materials is rather conveniently named metamaterials. While it is possible to create an artificial medium that possesses simultaneous negative permittivity and permeability, in a broader context one can use metamaterials to engineer a medium that has the desired electromagnetic response.

The transmission, refraction, and focusing properties of metamaterials have been extensively studied. Several researchers demonstrated negative permeability, left-handed transmission, negative refraction, and subwavelength focusing by using metamaterials. ${ }^{2,4}$ On the other hand, the emission properties of sources when they are placed inside metamaterials still remain to some extent unexplored. The study of the field emitted from a source, which is placed inside a left-handed medium slab, provides exciting answers for both physical and technological considerations. First of all, such a study provides the means of checking the validity of ray optics on left-handed medium metamaterials whose overall dimensions are comparable with one wavelength. In addition, the information gained from such studies is important for the applications that incorporate radiation sources and active or nonlinear materials inside left-handed medium metamaterials. ${ }^{7-11}$ There are several theoretical studies that investigated the issue of sources inside a metamaterial with permittivity and permeability equal to zero. In addition, there are some studies that attempted to use metamaterials to enhance the characteristics of antennas. ${ }^{12}$

In the present work, we studied the properties of the electromagnetic fields emitted from a source embedded inside a two-dimensional left-handed metamaterial that was based on labyrinth resonators. Our results showed that when the source is placed at a certain distance from the interface, the transmitted field is focused on subwavelength dimensions. We first present our retrieval results regarding the permittivity and permeability of the labyrinthbased left-handed medium. We show that there is a frequency at which the permittivity and permeability attain the same negative value, i.e., they are impedance matched to free space. We subsequently present our theoretical and experimental results for the impedance-matched frequency when the source is placed inside the left-handed medium.

The structure that we used for this present study was a two-dimensional periodic arrangement of wires and labyrinth structures. The details regarding the structure can be found elsewhere. ${ }^{13}$ We calculated the effective permittivity and permeability of the two-dimensional medium by using a retrieval procedure. The results are shown in Fig. 1. There is a certain frequency range at which the permittivity and permeability are negative. In addition, the values of permittivity and permeability (real parts) are the same at $6.3 \mathrm{GHz}$ with a value of -1.24 . Moreover, the

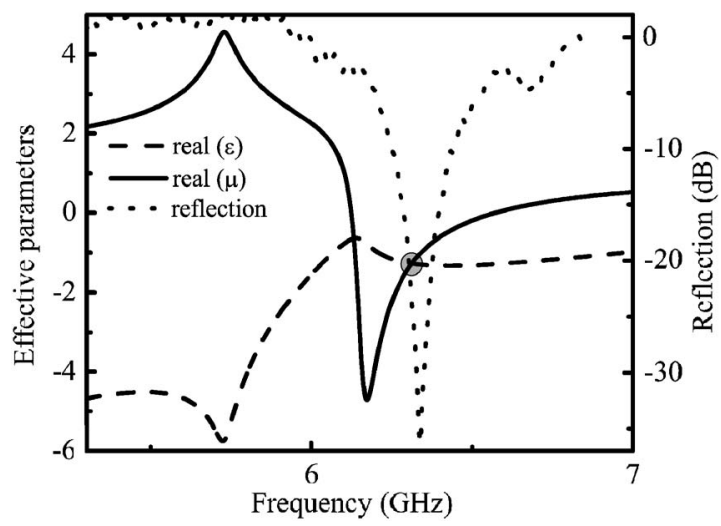

Fig. 1. Calculated effective medium parameters of the labyrinth-based left-handed medium: real part of $\epsilon$ (dashed) and real part of $\mu$ (solid). Measured reflection spectrum from the labyrinth-based left-handed medium (dotted). 
values of the imaginary parts are low at this frequency, 0.014 and 0.1 , respectively. As a result, the impedance of the medium is matched to the free space at $6.3 \mathrm{GHz}$. Therefore one expects a significant reduction of the reflection coefficient around $6.3 \mathrm{GHz}$. We measured the reflection spectrum from the twodimensional left-handed medium by using an HP8510C network analyzer. Receiving and transmitting horn antennas were used for the measurements. The measured reflection spectrum of the two-dimensional medium is shown in Fig. 1. The reflection from the two-dimensional medium is rather small around $6.31 \mathrm{GHz}(-35 \mathrm{~dB}$ at $6.31 \mathrm{GHz})$.

We carried out finite-difference time domain simulations to study the properties of the electromagnetic waves when the source was placed inside the lefthanded medium. In our simulations, we treated the two-dimensional labyrinth-based medium as a slab of homogeneous and isotropic material. This approximation provides the means to compare the actual left-handed metamaterial with an isotropic and homogeneous medium. In addition, such an approximation is justified because the size of the labyrinth features are very small compared with the wavelength of interest. The permittivity and permeability of the slab were set to -1.24 , and the simulation frequency was $6.3 \mathrm{GHz}$. The thickness of the slab was equal to the thickness of the labyrinth-based medium that we used in our experiments, $8.8 \mathrm{~cm}$ (10 layers of a labyrinth-based medium). Note that the thickness of the slab was $1.87 \lambda$. The source was placed seven layers, $6.16 \mathrm{~cm}$, away from the left side interface. The result of the finite-difference time domain simulation is shown in Fig. 2. When the source is seven layers away from the interface (left side interface), there is a clear focusing of the source field away from the interface. The half-width of the image along the $y$ axis at the maximum intensity point is $\lambda / 2.35$. On the other hand, when the source is three layers away from the interface (right side interface), the image is rather close to the surface. The half-width of the image in this case is reduced to $\lambda / 3.42$. It is noteworthy that geometrical optics considerations would lead image points away from the interfaces for both cases.

We used an HP-8510C network analyzer to perform the experiments. The transmitting monopole antenna was placed inside the two-dimensional labyrinth-based left-handed medium. The width of the labyrinth-based medium was ten layers, $8.8 \mathrm{~cm}$. The receiving monopole antenna was used to measure the field outside the left-handed medium over an area of $50 \mathrm{~mm} \times 100 \mathrm{~mm}$ area with steps of $2.5 \mathrm{~mm}$. More details regarding the experimental procedure can be found elsewhere. ${ }^{14}$ The results of our measurements when the source was three layers and seven layers away from the interface are shown in Figs. 3(a) and 3(b), respectively. The image of the source field appeared to be tied to the surface when the source was three layers away from the interface. The half-width of the image was $\lambda / 3.2$. On the other hand, the image appeared away from the interface when the source was placed inside the seventh layer. The maximum intensity point along the $x$ axis was
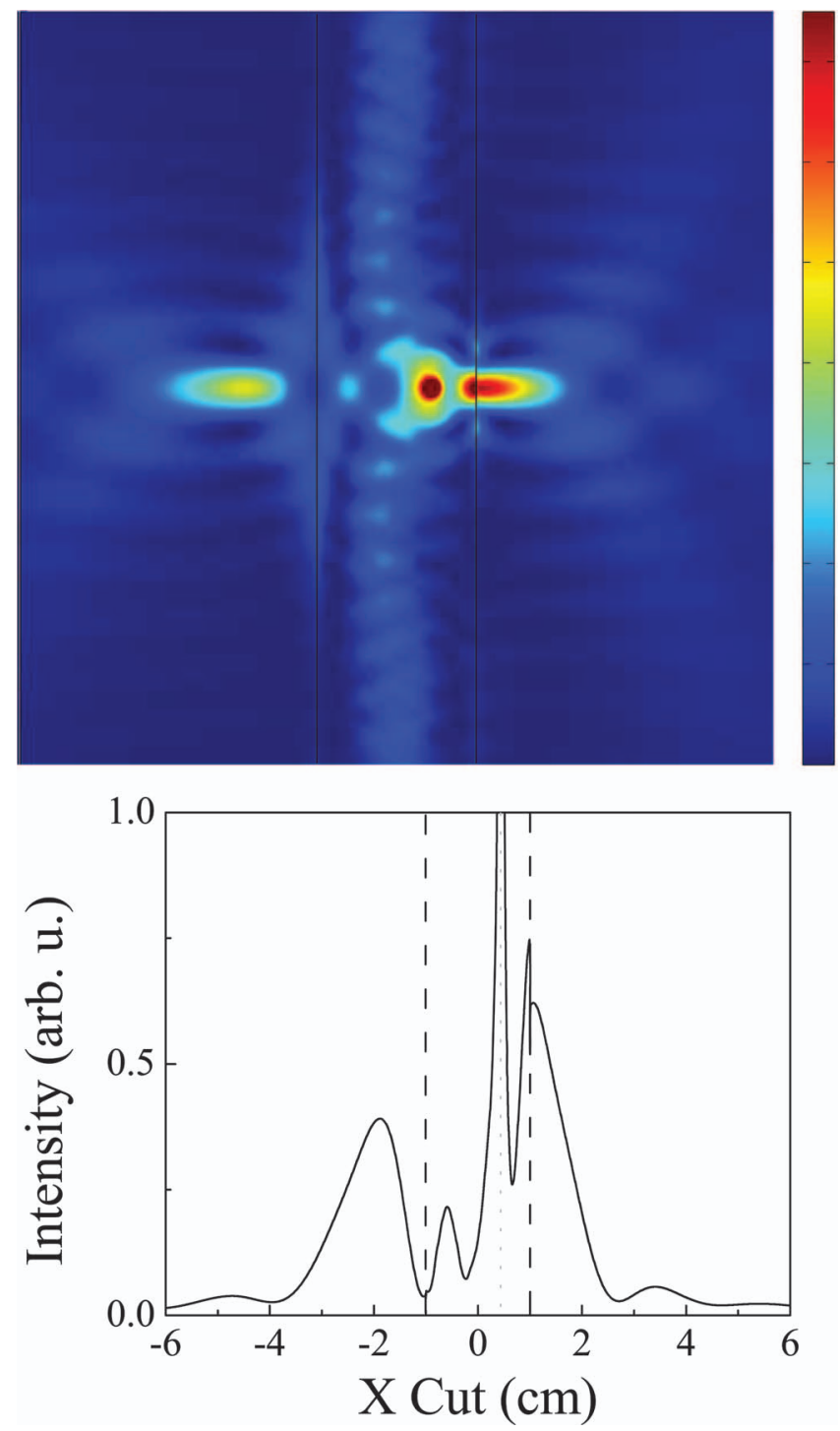

Fig. 2. Top, simulated field intensity emitted from a source placed inside the homogeneous left-handed medium. Bottom, cross section of the field intensity along the $x$ axis on the source plane.

$10 \mathrm{~mm}$ from the interface. In addition, the half-width of the image was $\lambda / 2.3$. We note that comparison of Figs. 2and 3 shows quite good agreement between our simulation results, which treated the left-handed medium as a homogenous and isotropic material, and experimental results. Both of these results indicate that one cannot treat a left-handed medium by use of geometrical optics if the overall dimensions of the medium are comparable with the wavelength of interest. The reason for this is that if we were to apply the argument of the geometrical optics, we would expect to observe an image away from the surface for both of the source distances. The discrepancy between both experimental and theoretical results and the expectations of geometrical optics is attributed to two main reasons. One reason is related to the size of the left-handed medium. As the size of the lefthanded medium is comparable with the wavelength, the results of geometrical optics are questionable. The second reason is related to the excitation of the surface plasmon waves. Figure 2 shows the existence 


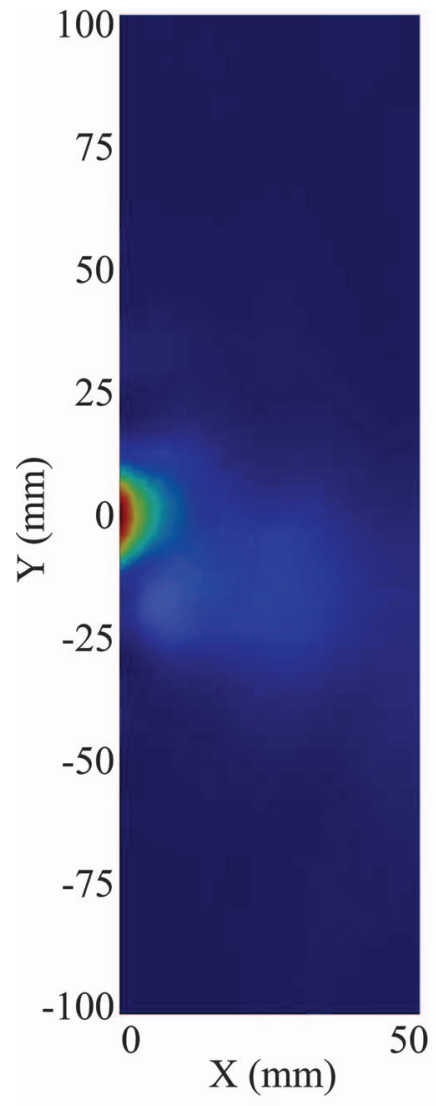

(a)

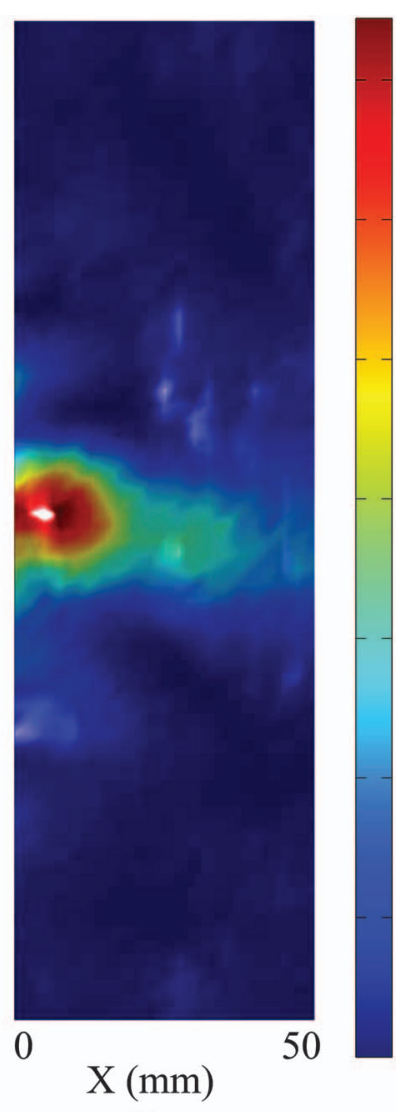

(b)
Fig. 3. Measured field intensity when the source was placed (a) three and (b) seven layers away from the interface inside the labyrinth-based left-handed medium.

of surface waves along the edges of the left-handed medium.

In conclusion, we studied the properties of the field emitted from a source that is embedded inside a twodimensional left-handed medium. Our results showed that when the source was at a certain distance away from the interface (seven layers in the present study), the image of the source appeared away from the interface. On the other hand, the image appeared tied to the surface when the source was placed closer to the interface. In addition, we observed half-widths of source field images as small as $\lambda / 3.7$.

I. Bulu's e-mail address is irfan@fen.bilkent.edu.tr.

\section{References}

1. V. G. Veselago, Sov. Phys. Usp. 10, 509 (1968).

2. D. R. Smith, W. J. Padilla, D. C. Vier, S. C. NematNasser, and S. Schultz, Phys. Rev. Lett. 84, 4184 (2000).

3. A. M. Belyantsev and A. B. Kozyrev, Tech. Phys. 47, 1477 (2002).

4. I. Bulu, H. Caglayan, and E. Ozbay, Opt. Lett. 31, 814 (2006).

5. P. Markos and C. M. Soukoulis, Phys. Rev. B 65, 033401 (2001).

6. J. B. Pendry, A. J. Holden, D. J. Robins, and W. J. Stewart, IEEE Trans. Microwave Theory Tech. 47, 2075 (1999).

7. R. W. Ziolkowski, Phys. Rev. E 70, 046608 (2004).

8. A. A. Zharov, I. V. Shadrivov, and Y. S. Kivshar, Phys. Rev. Lett. 91, 037401 (2003).

9. J. A. Porto, L. Martin-Moreno, and F. J. Garcia-Vidal, Phys. Rev. B 70, 081402 (2004).

10. A. Kafaratzis and Z. Hu, in IEEE High Frequency Postgraduate Student Colloquium (IEEE, 2005), p. 77.

11. J.-P. Xu, N.-H. Liu, and S.-Y. Zhu, Phys. Rev. E 73, 016604 (2006).

12. S. Enoch, G. Tayeb, P. Sabouroux, N. Guérin, and P. Vincent, Phys. Rev. Lett. 89, 213902 (2002).

13. I. Bulu, H. Caglayan, and E. Ozbay, Opt. Express 13, 10238 (2005).

14. I. Bulu, H. Caglayan, and E. Ozbay, Opt. Lett. 31, 814 (2006). 